\title{
Merger Simulation with Brand-Level Margin Data: Extending PCAIDS with Nests
}

\author{
Roy J. Epstein* \\ Daniel L. Rubinfeld ${ }^{\dagger}$
}

March 2004

(C) 2004 by Roy J. Epstein and Daniel L. Rubinfeld

This paper was originally published in Advances in Economic Analysis \& Policy, Vol. 4: No. 1, Article 2 (The B.E. Journals in Economic Analysis \& Policy), http://www.bepress.com/bejeap/advances/vol4/iss1/art2.

\footnotetext{
* Contact author, Boston College, Dept. of Finance. Email: rje@royepstein.com.

$\dagger$ University of California at Berkeley. Email: rubinfeld@law.berkeley.com.
} 


\section{INTRODUCTION}

Merger simulation has developed rapidly within the field of industrial organization as an important tool to evaluate unilateral price effects of mergers involving differentiated goods. ${ }^{1}$ Simulation typically calculates these effects as percentage changes in equilibrium prices between the pre- and post-merger markets, assuming the absence of overt collusion among competitors. A virtually unknown area a few years ago, the FTC has recently termed simulation among the past decade's "remarkable developments in the quantitative analysis of horizontal mergers." 2 The appeal of simulation is that it provides an economically coherent framework to quantify potential unilateral price increases, taking into account market shares, efficiencies, and other key features of a transaction.

In practice, simulation has to confront the significant practical constraints of the merger review process, including limited amounts of data, the need to control costs, and regulations that often permit only a short amount of time for the evaluation of competitive effects. Much research relating to merger simulation has focused on other complications involving the appropriate specification of demand systems, the empirical estimation of parameters, the assumption of static versus dynamic pricing behavior, and other methodological issues. ${ }^{3}$ Given these factors, there is increasing interest in methods to obtain values for the inputs to the analysis that can make simulation a feasible and persuasive option in a broader range of situations. ${ }^{4}$

In this article we describe a modeling strategy to achieve these goals that is fundamentally different from existing approaches that are based on structural econometric estimation. ${ }^{5}$ Specifically, we integrate the PCAIDS (ProportionalityCalibrated Almost Ideal Demand System) merger simulation methodology (Epstein and Rubinfeld, 2002) with brand-level profit margin data. We recognize that relevant margin data at this level of detail are seldom available in general academic research. However, merger simulation is used by the antitrust agencies,

${ }^{1}$ For a recent review of some of the relevant literature, including references to the work of Werden and Froeb; Berry, Levinsohn, and Pakes; and Hausman, Leonard, and Zona, see Baker and Rubinfeld (1999).

${ }^{2}$ See Issues in Econometric Analysis of Scanner Data, available at www.ftc.gov.

${ }^{3}$ See Pinske and Slade (forthcoming) for a more detailed discussion.

${ }^{4}$ Werden and Froeb (2002).

${ }^{5}$ See, e.g., Berry, Levinsohn, and Pakes (1995) in addition to Pinske and Slade (forthcoming). 
which have the authority to request information from the merging parties (and possibly also from third parties). For this reason, margin data are likely to be available in actual merger reviews and we think it likely that such data will receive increasing scrutiny in the future. So while merger simulation that relies on margin data may be seen as addressing a narrow issue, it has potentially broad relevance for improving antitrust enforcement policy.

Our approach uses the margin data to estimate the PCAIDS parameters that define "nests" (i.e., groups of products that are particularly close substitutes). The nests provide a flexible and relatively parsimonious structure for estimating premerger demand elasticities that are consistent with the observed margins and that satisfy the Bertrand first-order conditions for profit maximization conventionally used in merger simulation. When reasonably accurate margin data are available and detailed econometric estimation is not feasible, one can use the PCAIDS approach with nests along with a relatively sophisticated model of demand to analyze the competitive effects of mergers.

Briefly, PCAIDS is an approximation to the Almost Ideal Demand System (AIDS) that is widely used in applied microeconomics. ${ }^{6}$ PCAIDS relies on a generalized principle of proportionality to reduce greatly the number of free parameters in the demand model: a price increase for a single brand results in diversion of lost sales to the other brands in proportion to their current market shares. In its most basic form (i.e., with all brands in a single nest), PCAIDS can be fully specified with two parameters: the margin for a single brand and the price elasticity for the market as a whole. If the market is well characterized by proportionality, this specification will yield a close approximation to the elasticities from the unrestricted AIDS. When the actual pattern of demand deviates from "strict" proportionality, the approximation can be improved by adding additional nests to generalize the analysis. ${ }^{7}$ As the amount of a priori margin (or elasticity) information increases, PCAIDS is able to calibrate more and more nests empirically. The resulting elasticities are less constrained than those implied by strict proportionality and can result in a much closer approximation to a full AIDS model.

The key to our analysis is the linkage between the "nesting parameters" in PCAIDS and the accounting data on profit margins. ${ }^{8}$ We will show that in some

\footnotetext{
${ }^{6}$ Deaton and Muellbauer (1980).

${ }^{7}$ Nests in our analysis are analogous to the different levels of a multi-stage budgeting model, an approach that is sometimes used to make econometric estimation tractable.

${ }^{8}$ The term "nesting parameter" will be used in place of "odds ratio factor" and "scaling factor" used in Epstein and Rubinfeld (2002), pp. 896, 897.
} 
circumstances the margin data are sufficient to identify the nesting parameters exactly, while in other cases the nesting parameters are not fully identified. Lack of identification is not necessarily a serious issue because the structure of the model nonetheless yields bounds on the possible values for the nesting parameters. In still other cases (corresponding roughly to a situation of many margins and relatively few nests) the nesting parameters will be overidentified, for which we describe additional calibration strategies.

Properly measured accounting margins hold out the prospect of more accurate model calibration compared to conventional econometric modeling of pre-merger own and cross-price elasticities.' The econometric approach is not well suited for many transactions because of a lack of adequate data even when strong assumptions are made about market structure (such as a multi-stage budgeting process) to reduce the large number of parameters to be estimated. Even with relatively large datasets, the empirical results can be problematic, with wrong signs, implausible magnitudes, and low statistical reliability for the estimated coefficients.

The balance of this article is organized as follows. In Section II we set the stage by briefly outlining the structure of PCAIDS with nests. Readers familiar with the analysis presented in Epstein and Rubinfeld (2002) can skip this review. In Section III we explain how accounting profit margin data can be used to infer nesting parameters empirically. Section IV briefly discusses some of the practical difficulties in interpreting accounting data in this context. Section V presents several examples of the analysis. Section VI contains a brief conclusion.

\section{Merger Simulation With PCAIDS}

Merger simulation models for differentiated products typically assume that prices in the market can be analyzed using Bertrand assumptions. According to this theory, the first-order conditions ("FOCs") for profit maximization by each firm can be specified in terms of market shares, incremental profit margins, and price elasticities. The market is assumed to be in Bertrand equilibrium both pre- and post-merger.

There are $n$ firms pre-merger. The $i$ th firm produces $n_{\mathrm{i}}$ brands and there is a total of $N$ brands in the market. A matrix expression for all of the FOCs for profit maximization is given by

${ }^{9}$ Werden and Froeb (2002) have suggested using margins to calibrate certain parameters in non-nested logit models. Epstein and Rubinfeld (2002) also provide an example of using margins to calibrate a non-nested PCAIDS model. 


$$
s+\operatorname{diag}\left(E_{1}, E_{2}, \ldots, E_{n}\right) S \mu=0 .
$$

In equation (1), $s=\left(s_{1}, s_{2}, \ldots s_{\mathrm{N}}\right)^{\prime}$ is the vector of market shares (in terms of revenue) and $S=\operatorname{diag}(s)$. The corresponding vector of brand margins is $\mu=\left(\mu_{1}\right.$, $\left.\mu_{2}, \ldots, \mu_{\mathrm{N}}\right)^{\prime}$. For the ith firm, $E_{\mathrm{i}}$ is an $n_{\mathrm{i}}$ by $n_{\mathrm{i}}$ matrix of transposed price elasticities with element $(\mathrm{k}, \mathrm{j})$ equal to $\varepsilon_{\mathrm{jk}}$. In the pre-merger equilibrium, the brand margins $\mu$ are given by

$$
\mu=-S^{-1} \operatorname{diag}\left(E_{1}, E_{2}, \ldots, E_{n}\right)^{-1} s .
$$

Assume that the merger involves firms 1 and 2. The merged firm is characterized by an augmented elasticity matrix $E^{*}$ for the $n_{1}+n_{2}$ brands it is now producing. The FOCs for the post-merger market are

$$
s+\operatorname{diag}\left(E^{*}, E_{3}, \ldots, E_{n}\right) S \mu=0,
$$

where all variables are understood to be taken at their post-transaction values. Merger simulation consists of finding the post-merger prices that yield margins, shares, and elasticities that solve (3).

The solution to equation (3) depends on the functional form of the underlying demand model and a supply model that determines how total cost responds to incremental changes in post-merger output. The demand side can be modeled using a variety of specifications; the literature includes examples of linear, constant elasticity, and variants of logit and AIDS systems. The supply side is generally treated as a step-function for which incremental cost does not vary with output. The step allows for possible merger-specific efficiencies, which are analyzed by changing the level of post-merger incremental costs (keeping the assumption that the new level of incremental cost does not vary with output).

In PCAIDS each $s_{\mathrm{i}}$ is a linear function of the natural logarithms of all of the brands in the relevant market. Letting $p$ be the vector of brand prices, the model can be written as $s=a+B \ln (p)$, where $a$ is a vector of constants and $B$ is a matrix of coefficients (that are assumed to be invariant to price changes). Unlike AIDS, PCAIDS does not include an aggregate expenditure term in the share equations. ${ }^{10}$ To proceed, differentiate each share equation totally to obtain:

$$
\mathrm{d} s=B(\mathrm{~d} p / p)
$$

\footnotetext{
${ }^{10}$ Though lacking the expenditure term, the PCAIDS brand price elasticities in general are different from AIDS price elasticities that impose homotheticity (compare equations (5) and (6) to the AIDS elasticities in Alston, Foster and Green, 1994). Further research on the empirical implications of alternative model specifications that depart from homotheticity would be worthwhile.
} 
Equation (4) describes a simple relationship between the change in each brand's market share $(\mathrm{d} s)$ and the unilateral effects $(\mathrm{d} p / p)$. The elements $b_{\mathrm{ij}}$ of $B$ act as weights to determine the amount of share lost or gained due to unilateral effects. Moreover, as is apparent from (4), knowledge of the $a_{\mathrm{i}}$ terms is unnecessary. The post-merger shares for use in equation (3) are given by $s^{\text {post }}=s^{\mathrm{pre}}+\mathrm{d} s$. PCAIDS therefore is a particularly convenient demand model for merger simulation.

The post-merger own and cross-price elasticities for each brand in the market in general will also depend on the vector $\mathrm{d} p / p$ of unilateral effects. It can be shown ${ }^{11}$ that in PCAIDS:

Own-price elasticity for the ith brand: $\mathcal{E}_{\mathrm{ii}}=-1+\frac{b_{\mathrm{ii}}}{s_{\mathrm{i}}}+s_{\mathrm{i}}(\varepsilon+1)$

Cross-price elasticity of the ith brand with respect to the price of the jth

brand: $\varepsilon_{\mathrm{ij}}=\frac{b_{\mathrm{ij}}}{s_{\mathrm{i}}}+s_{\mathrm{j}}(\varepsilon+1)$.

Here $\varepsilon$ is the price elasticity of demand for the market as a whole, which is typically assumed to remain unchanged post-merger. Using the $s^{\text {post }}$ vector in equations (5) and (6) yields the post-merger elasticities.

Finally, the solution to (3) requires updated brand profit margins. Algebraically, it can be seen that for each brand, $\mu_{\mathrm{i}}^{\mathrm{post}}=1-\left(1-\mu_{\mathrm{i}}^{\mathrm{pre}}\right) / \exp \left(\mathrm{d} p_{\mathrm{i}} / p_{\mathrm{i}}\right)$. This relationship is independent of the demand model. This structure is sufficient to solve the post-merger FOCs entirely in terms of the predicted unilateral effects $\mathrm{d} p / p .^{12}$

\section{A. Calibrating PCAIDS Under Strict Proportionality}

The problem remains of finding appropriate values for the $b_{\mathrm{ij}}$. PCAIDS assumes that the share lost as a result of a price increase is allocated to the other firms in the relevant market in proportion to their respective shares. In effect, the market shares define probabilities of making incremental sales for each of the competitors. We also impose homogeneity on the demand model in equation (4), i.e., $\sum b_{\mathrm{ik}}=0 \forall \mathrm{k}$. (Since shares must sum to $100 \%$, the model also satisfies an adding-up constraint $\sum b_{\mathrm{ki}}=0 \quad \forall \mathrm{k}$ by definition). Homogeneity with the proportionality assumption implies symmetry of $B$, thereby satisfying Slutsky symmetry, as will be proved below.

${ }^{11}$ Epstein and Rubinfeld (2002), Appendix.

${ }^{12}$ For additional structure to take account of merger-related efficiencies in PCAIDS, see $i d$. 
A three-brand example will illustrate the basic proportionality assumption. Consider a price increase $\mathrm{d} p_{1} / p_{1}$ with all other prices unchanged. With proportionality, sales are diverted to brands 2 and 3 in proportion to their market shares. That is, $\mathrm{d} s_{2} / \mathrm{d} s_{3}=s_{2} / s_{3}$. Moreover, the sum of the changes in shares across all brands must equal zero (because shares must always sum to $100 \%$ ). It follows that $b_{21}$ equals $-s_{2} /\left(s_{2}+s_{3}\right) b_{11}$ and $b_{31}$ equals $-s_{3} /\left(s_{2}+s_{3}\right) b_{11}$ (the minus sign is necessary to satisfy $\left.\sum \mathrm{d} s_{\mathrm{i}}=0\right)$. The other coefficients in $B$ can be calibrated similarly, e.g., a change $\mathrm{d} p_{2} / p_{2}$ implies $b_{12}$ equals $-s_{1} /\left(s_{1}+s_{3}\right) b_{22}$ and $b_{32}$ equals $s_{3} /\left(s_{1}+s_{3}\right) b_{22}$. $B$ can accordingly be expressed in this example as:

$$
B=\left(\begin{array}{lll}
b_{11} & \frac{-s_{1}}{\left(s_{1}+s_{3}\right)} b_{22} & \frac{-s_{1}}{\left(s_{1}+s_{2}\right)} b_{33} \\
\frac{-s_{2}}{\left(s_{2}+s_{3}\right)} b_{11} & b_{22} & \frac{-s_{2}}{\left(s_{1}+s_{2}\right)} b_{33} \\
\frac{-s_{3}}{\left(s_{2}+s_{3}\right)} b_{11} & \frac{-s_{3}}{\left(s_{1}+s_{3}\right)} b_{22} & b_{33}
\end{array}\right) .
$$

The matrix is completely determined by the three unknown diagonal elements. Proportionality has dramatically reduced the calibration problem from order $N^{2}$ to $\operatorname{order} N$.

Homogeneity and adding-up further simplify the problem. Express $b_{33}$ as $b_{31}-b_{32}$ and substitute into $b_{11}=-b_{12}-b_{13}$ to find $b_{22}=\left(s_{2} / s_{1}\right)\left(1-s_{2}\right) /\left(1-s_{1}\right) b_{11}$. Similarly, $b_{33}=\left(s_{3} / s_{1}\right)\left(1-s_{3}\right) /\left(1-s_{1}\right) b_{11}$. That is, the entire demand model under proportionality can be calibrated in terms of a single parameter. (We prove below that this result holds regardless of the number of brands in the market.) Assuming the own-price elasticity $\varepsilon_{11}$ is known for the first brand and that the market elasticity of demand $\varepsilon$ is also known, invert equation (5) to find $b_{11}=s_{1}\left(\varepsilon_{11}+1-\right.$ $s_{1}(\varepsilon+1)$ ). The $B$ matrix is then determined by appropriate scaling of $b_{11}$ with the market shares.

These ideas can be illustrated as follows. Assume that the shares for the 3 brands (each sold by a different firm) are $20 \%, 30 \%$, and 50\%, respectively. Now, assume that there is a proposed merger between firms 1 and 2, the industry elasticity is -1 , and the own-price elasticity for the first brand is -3 . Table 1 shows the resulting $B$ matrix and elasticities. 


\section{Table 1}

\section{PCAIDS Coefficients and Elasticities}

\begin{tabular}{|c|c|c|c|c|c|c|}
\hline$\underline{\text { Brand }}$ & \multicolumn{3}{|c|}{$\underline{\text { PCAIDS Coefficient Matrix }}$} & $p_{1}$ & $\underline{p}_{2}$ & $\underline{p}_{3}$ \\
\hline 1 & -0.400 & 0.150 & 0.250 & -3.00 & 0.75 & 1.25 \\
\hline 2 & 0.150 & -0.525 & 0.375 & 0.50 & -2.75 & 1.25 \\
\hline 3 & 0.250 & 0.375 & -0.625 & 0.50 & 0.75 & -2.25 \\
\hline
\end{tabular}

The PCAIDS coefficients satisfy adding-up and homogeneity and are symmetric, as required.

Table 1 illustrates an important feature of strict proportionality: it constrains the cross-price elasticities corresponding to a given price change to be equal, although they may still vary substantially with respect to price increases across brands. ${ }^{13}$ The ability to derive a large number of elasticities from a single parameter (e.g., $b_{11}$ ) therefore comes at the expense of some flexibility in the model. This constraint is the main difference between PCAIDS and the full, unrestricted AIDS. It is generally of most concern when products are highly differentiated, since proportionality may not accurately describe the diversion of sales in those circumstances. ${ }^{14}$ The purpose of nests is to relax this constraint and allow a closer approximation to the unrestricted AIDS.

\section{B. Nests and Deviations from Strict Proportionality}

We allow a more general analysis of elasticity by grouping brands in "nests." 15 Proportionality governs diversion within a nest, where brands are relatively close substitutes. Brands are poorer substitutes across nests than indicated by proportionality, implying variation in the cross-price elasticities. While $\varepsilon_{\mathrm{ik}}=\varepsilon_{\mathrm{jk}}$

${ }^{13}$ This equality of the cross-price elasticities is sometimes referred to as "independence of irrelevant alternatives" or IIA.

${ }^{14}$ Cf. Horizontal Merger Guidelines at 92.211 : “The market shares of the merging firms' products may understate the competitive effect of concern, when, for example, the products of the merging firms are relatively more similar in their various attributes to one another than to other products in the relevant market. On the other hand, the market shares alone may overstate the competitive effects of concern when, for example, the relevant products are less similar in their attributes to one another than to other products in the relevant market."

${ }^{15}$ See Werden and Froeb (1994) for a discussion of nests in the context of a logit demand model. 
for brands in the same nest, the cross-price elasticities are (relatively) lower across nests, i.e., $\varepsilon_{\mathrm{mk}}<\varepsilon_{\mathrm{ik}}$ for brands $\mathrm{m}$ and i in different nests.

To illustrate the analysis of proportionality with nests, return to the threebrand example discussed in the previous section. In that example, brand 2's market share of $30 \%$ and brand 3's share of $50 \%$ implied that $37.5 \%(30 / 80)$ of the share lost by brand 1 when its price increased would be diverted to brand 2 and $62.5 \%(50 / 80)$ would be diverted to brand 3 . This effect can be characterized using an odds ratio. Here, the odds ratio between brand 2 and brand 3 is 0.6 $(0.375 / 0.625)$. That is, the probability that consumers switch to brand 2 is $60 \%$ of the probability that they would switch to brand 3. Now suppose instead that brand 2 is relatively "farther" from brand 1 in the sense that fewer consumers would choose brand 2 in response to an increase in $p_{1}$ than would be predicted by proportionality. For example, brand 2 may only be "half as desirable" a substitute as brand 3 so that the appropriate odds ratio is really only 0.3 . It is straightforward to calculate in this case that the share diversion to brand 2 becomes $23.1 \%$ and the diversion to brand 3 increases to $76.9 \%$ (odds ratio of 0.3 $=.231 / .769)$. As expected, fewer consumers leaving brand 1 would choose brand 2.

We use "nesting parameters" to adjust diversion away from proportionality. Share diverted to a brand in a different nest is adjusted in the following sense: the odds ratio is equal to the odds ratio under proportionality, multiplied by a nesting parameter on the interval $(0,1]$. For brands within a nest, the nesting parameter equals 1 . The result is that brands within a nest are closer substitutes than brands outside the nest. Proportionality for all brands can be thought of as a model with a single nest. PCAIDS with multiple nests allows a more flexible pattern of cross elasticities, as the model is no longer fully constrained by the proportionality assumption. ${ }^{16}$

To characterize the nest structure in the above example we place brand 2 in a separate nest with a nesting parameter of 0.5 . Table 2 reports the calculated elasticities for both the nested model and the original model.

${ }^{16}$ For example, Wu (2003) provides an example criticizing the flexibility of PCAIDS. However, his analysis does not use nests, which as we show here, serves to greatly generalize the model. 
Table 2

\section{PCAIDS Elasticities with Nests}

\begin{tabular}{|c|c|c|c|c|c|c|}
\hline \multirow[b]{3}{*}{ Brand } & \multirow{2}{*}{\multicolumn{3}{|c|}{$\begin{array}{l}\text { Non-Nested Demand } \\
\text { Elasticity with Respect to: }\end{array}$}} & \multicolumn{3}{|c|}{$($ Nesting Parameter $=0.5)$} \\
\hline & & & & Ela & with $\mathrm{R}$ & t to: \\
\hline & $p_{1}$ & $\underline{p}_{2}$ & $\underline{p}_{3}$ & $p_{1}$ & $p_{2}$ & $p_{3}$ \\
\hline 1 & -3.00 & 0.75 & 1.25 & -3.00 & 0.46 & 1.54 \\
\hline 2 & 0.50 & -2.75 & 1.25 & 0.31 & -2.08 & 0.77 \\
\hline 3 & 0.50 & 0.75 & -2.25 & 0.62 & 0.46 & -2.08 \\
\hline
\end{tabular}

The nest has a variety of effects. The cross-price elasticities for brand 2 in the right-hand panel are scaled down by $50 \%$ relative to the other brands. That is, IIA no longer holds (the cross elasticities $\varepsilon_{12}$ and $\varepsilon_{32}$ remain equal because brands 1 and 3 are in the same nest). The nest implies diminished interbrand competition, as reflected by the smaller own-price elasticities for brands 2 and 3. However, brands 1 and 3 become relatively more substitutable on the basis of cross-price elasticity. ${ }^{17}$

The number of nesting parameters required in the model obviously depends on the number of nests. More specifically, the number of parameters equals the number of pairs of nests, because each parameter modifies the share diversion between the two associated nests. With 2 nests there is one nesting parameter; a 3-nest specification requires three parameters; and a 4-nest specification requires six parameters. Because the number of nesting parameters increases exponentially with the number of nests, a tractable simulation model probably should not have more than 3 or 4 nests.

Assume that there are $w$ nests, $2 \leq w \leq N$, with each brand assigned to a nest. There are $w(w-1) / 2$ distinct nesting parameters, denoted $\omega_{1}$. It is helpful to arrange them in a symmetric $w$ by $w$ matrix. In the case of three nests the matrix takes the form

$$
\Omega=\left(\begin{array}{lll}
100 \% & \omega_{1} & \omega_{2} \\
\omega_{1} & 100 \% & \omega_{3} \\
\omega_{2} & \omega_{3} & 100 \%
\end{array}\right)
$$

\footnotetext{
${ }^{17}$ The calculations continue to assume an own-price elasticity of -3 for Brand 1 and an industry elasticity of -1 . It would be incorrect to scale the non-nested elasticities in the left-hand panel directly. Nests affect the impact of adding-up, homogeneity, and symmetry, and the appropriate calculation takes account of these constraints to generate economically consistent elasticities.
} 
Given a price increase for brand $\mathrm{i}$, the diversion of share to a brand $\mathrm{j}$ deviates from proportionality by the nesting parameter $0<\Omega(\mathfrak{I}(\mathrm{i}), \mathfrak{I}(\mathrm{j})) \leq 1$. $\mathfrak{I}(\mathrm{k})$ is an indicator function that returns the nest containing brand $\mathrm{k}$. For example, if brand $\mathrm{i}$ were in nest 2 and brand $\mathrm{j}$ were in nest 1, the appropriate nesting parameter would be $\omega_{1}$. Proportionality is the special case where $\Omega(\mathfrak{J}(\mathrm{i}), \mathfrak{I}(\mathrm{j}))=100 \%$. The diagonal elements of $\Omega$ equal $100 \%$ since proportionality is assumed to hold within a given nest.

We illustrate a general calculation of $b_{\mathrm{ij}}$ with nesting parameters with the example used for Table 2. There are two nests, so $\Omega$ contains a single subdiagonal element $\omega_{1}$. We assume that $\Omega(\mathfrak{I}(1), \mathfrak{I}(2))=\omega_{1}$ and that $\Omega(\mathfrak{I}(1), \mathfrak{I}(3))=$ $100 \%$. That is, brands 1 and 3 are in a common nest and brand 2 is in a separate nest. The share diversion for the price change for brand 1 can be expressed (imposing the adding-up condition) as

$$
-\mathrm{d} s_{1}+\lambda_{2} s_{2} /\left(1-s_{1}\right) \mathrm{d} s_{1}+\lambda_{3} s_{3} /\left(1-s_{1}\right) \mathrm{d} s_{1}=0
$$

or, rewriting,

$$
\lambda_{2} S_{2} /\left(1-S_{1}\right)+\lambda_{3} S_{3} /\left(1-S_{1}\right)=1
$$

where the $\lambda$ 's are share-diversion weights to be determined. In the case of strict proportionality, $\lambda_{i}=100 \%$. The adjustment due to the deviation from proportionality in this example satisfies $\lambda_{2} / \lambda_{3}=\Omega(\mathfrak{I}(1), \mathfrak{I}(2)) / \Omega(\mathfrak{I}(1), \mathfrak{I}(3))=$ $\omega_{1}$. Therefore $\lambda_{2}=\omega_{1} \lambda_{3}$ and by equation (9), $\lambda_{3}=\left(1-s_{1}\right) /\left(\omega_{1} s_{2}+s_{3}\right)$.

By substitution, equation (8) yields $-\mathrm{d} s_{1}+\omega_{1} s_{2} /\left(\omega_{1} s_{2}+s_{3}\right) \mathrm{d} s_{1}+s_{3} /\left(\omega_{1} s_{2}+\right.$ $\left.s_{3}\right) \mathrm{d} s_{1}=0$. That is, $b_{21}=\omega_{1} s_{2} /\left(\omega_{1} s_{2}+s_{3}\right) b_{11}$ and $b_{31}=s_{3} /\left(\omega_{1} s_{2}+s_{3}\right) b_{11}$. To evaluate these expressions substitute the shares $(30 \%, 50 \%), b_{11}=-0.400$ (unchanged from Table 1), and $\omega_{1}=0.5$. The results are 0.231 and 0.769 , agreeing with the share diversion percentages that we calculated at the beginning of this section.

\section{General Calibration of PCAIDS with Nests}

We generalize the determination of $B$ with nests as follows. Each element of $B$ can be written as $b_{\mathrm{ik}}=\theta_{\mathrm{ik}} b_{\mathrm{kk}}$, where the $\theta$ s are known, but the diagonal elements $b_{\mathrm{kk}}$ are unknown. Impose adding-up and homogeneity. The constraints imply a system of $N-1$ independent equations in the $N$ unknown diagonal elements. Without loss of generality, order the system so that $b_{11}$ corresponds to the first brand with a known margin. We normalize with respect to that brand and define a vector $\beta$ with $N-1$ elements equal to $b_{\mathrm{jj}} / b_{11}=\beta_{\mathrm{j}}, \mathrm{j}>1$. The equation system is then non-singular and can be written as 


$$
\left(\begin{array}{llll}
\theta_{12} & \theta_{13} & \ldots & \theta_{1 N} \\
1 & \theta_{23} & \cdots & \theta_{2 N} \\
\vdots & & & \\
\theta_{N-1,2} & \cdots & 1 & \theta_{N-1, N}
\end{array}\right)\left(\begin{array}{l}
\beta_{2} \\
\vdots \\
\beta_{N}
\end{array}\right)=\left(\begin{array}{l}
-1 \\
-\theta_{21} \\
\vdots \\
-\theta_{N-1,1}
\end{array}\right)
$$

Equation (10) can be inverted to solve for the $\beta$ vector. $B$ can therefore be specified entirely in terms of $b_{11}$ and the $\theta$ s. With known $\varepsilon_{11}$ and $\varepsilon$, calibration is completed by using (5) to solve for the value for $b_{11}$.

The $\theta$ s are known functions of the market shares and the nesting parameters. In general it can be shown that:

$$
\theta_{\mathrm{ik}}=-s_{\mathrm{i}} \frac{\Omega(\mathfrak{I}(\mathrm{k}), \mathfrak{I}(\mathrm{i}))}{\sum_{\mathrm{m} \neq \mathrm{k}} s_{\mathrm{m}} \Omega(\mathfrak{I}(\mathrm{k}), \mathfrak{I}(\mathrm{m}))}, \mathrm{i} \neq \mathrm{k} .
$$

With strict proportionality (i.e., a single nest that contains all of the brands), the nesting parameter equals $100 \%$ and equation (11) reduces to $\theta_{\mathrm{ik}}=-s_{\mathrm{i}} /\left(1-s_{\mathrm{k}}\right)$.

We now show that the matrix $B$ of PCAIDS coefficients is symmetric both under strict proportionality and with nests. Since equation (10) has a unique solution, any feasible solution is also unique. We try the symmetric solution for $B$ so that $b_{\mathrm{j} 1}=\theta_{\mathrm{j} 1} b_{11}=b_{1 \mathrm{j}}=\theta_{1 \mathrm{j}} b_{\mathrm{j} j}$, implying $\beta_{\mathrm{j}}=\theta_{\mathrm{j} 1} / \theta_{1 \mathrm{j}}$. From equation (11) it follows that

$$
\beta_{\mathrm{j}}=\frac{s_{\mathrm{j}}}{s_{1}} \frac{\sum_{\mathrm{m} \neq \mathrm{j}} s_{\mathrm{m}} \Omega(\mathfrak{I}(\mathrm{j}), \mathfrak{I}(\mathrm{m}))}{\sum_{\mathrm{m} \neq 1} s_{\mathrm{m}} \Omega(\mathfrak{J}(1), \mathfrak{I}(\mathrm{m}))}
$$

and from before, $b_{\mathrm{jj}}=\beta_{\mathrm{j}} b_{11}$.

Equations (11) and (12) imply that

$$
b_{\mathrm{ij}}=-\frac{s_{\mathrm{i}} s_{\mathrm{j}}}{s_{1}} \frac{\Omega(\mathfrak{I}(\mathrm{i}), \mathfrak{J}(\mathrm{j}))}{\sum_{\mathrm{k}=2}^{N} s_{\mathrm{k}} \Omega(\mathfrak{I}(\mathrm{k}), \mathfrak{I}(1))} b_{11} .
$$

for $\mathrm{i} \neq \mathrm{j}$. Symmetry of $B$ follows directly. ${ }^{18}$ It can be shown that the $b_{\mathrm{ij}}$ from equation (13) satisfy adding-up and homogeneity. They therefore comprise the unique solution to equation (10).

${ }_{18}$ The marketing literature offers mixed empirical support for symmetry restrictions when dealing with narrowly defined and highly differentiated products; see Blattberg and Wisniewski (1989). Imposing symmetric assumptions when they do not hold will affect the merger simulation results. 
Readers may wonder whether the nesting analysis is suited to study mergers at the wholesale as well as retail level. In our opinion, PCAIDS can be applied to both kinds of markets. At the retail level, PCAIDS can be calibrated with market shares and demand elasticities of end-users (see Epstein and Rubinfeld, 2002). At the wholesale level, PCAIDS can be calibrated with market shares and margins. In both cases, the simulation can be carried out with a minimum of data and should be a valuable complement to other analyses based, for example, on the HHI. But when applying merger simulation to wholesale markets, one must think carefully before accepting the Bertrand pricing assumption. For example, a Bertrand equilibrium determined by continuously differentiable first-order conditions may not adequately describe wholesale markets where a large fraction of output is sold in a small number of "winner take all" auctions. Further research is required to explore the implication of these issues more completely.

\section{MARGINS AND Nests IN PCAIDS}

In this section we show how margin data can be used to estimate nesting parameters. Each firm in the market may sell brands in different nests and every brand must be assigned to a nest. As a theoretical matter, since proportionality is unlikely to hold exactly, each brand might be put in a separate nest. However, as previously discussed, this strategy rapidly generates an intractable number of required parameters. A priori information must be available to group brands more broadly. To simplify the analysis, we aggregate the brands in the same nest for a given firm into a composite brand whose share is equal to the sum of the shares of the underlying brands. That is, the number of different nests that a firm sells into equals the number of (possibly composite) brands that it produces.

As before, the merger involves firms 1 and 2. We assume that the margins are known for each of the brands they produce. Each nest has either one or two margins associated with it; one margin if only one firm sells a brand in the nest, and two otherwise. To further simplify, assume also that no other margins are known and that the industry elasticity equals -1 (these assumptions are not essential to the results that follow). From equations (5) and (12), it can be seen that the elasticity matrices $E_{\mathrm{i}}$ in the FOCs are functions of $b_{11}$, the market shares (and industry elasticity), and the unknown nesting parameters.

PCAIDS can introduce demand asymmetry by allowing $\Omega$ to be asymmetric, but the resulting increase in the number of nesting parameters is likely to make the approach intractable unless substantial prior information is available to calibrate the model. 
The problem of determining nesting parameters amounts to finding values for them that generate $E$ matrices that satisfy the FOCs with the pre-determined elements of the margin vector $\mu{ }^{19}$ The nesting parameters will depend only on the FOCs for the two merger partners because only those margins are known (more generally, the parameters will depend on the FOC equations for each firm with known margins). The structure of PCAIDS leads to a simple solution.

For notation, let a superscript ${ }^{*}$ denote the components of the FOCs relevant to the merging firms. Let $E^{*}=\operatorname{diag}\left(E_{1}, E_{2}\right)$, the transposed block-diagonal matrix of pre-merger brand elasticities. Let $B^{*}$ be the corresponding $n_{1}+n_{2}$ blockdiagonal matrix of coefficients from the $B$ matrix of PCAIDS coefficients. It is straightforward to show that $E^{*}=B^{*}\left(S^{*}\right)^{-1}-I^{*}$, where $S^{*}$ is the diagonal matrix of corresponding brand shares and $I^{*}$ is a conformable identity matrix. Finally, $\mu^{*}$ is the vector of known margins and $s^{*}$ is the vector of shares. The relevant implied margins from equation (2) can be expressed as

$$
\left.m^{*}=-\left(S^{*}\right)^{-1}\left[B^{*}\left(S^{*}\right)^{-1}-I^{*}\right)^{-1}\right]^{-1} S^{*} .
$$

The next step is to express $B^{*}$ in equation (14) in terms of the nesting parameters. Using equations (12) and (13), it follows that

$$
B^{*}=\left[-S^{*}\left(s^{*}\right)^{\prime} \otimes(\omega-I)+\operatorname{diag}\left(S_{\mathrm{ii}}^{*} \sum_{\mathrm{m} \neq \mathrm{i}} S_{\mathrm{m}} \Omega(\mathfrak{I}(\mathrm{i}), \mathfrak{J}(\mathrm{m}))\right)\right] \times \tau
$$

where

$$
\tau=\frac{b_{11}}{s_{1} \sum_{\mathrm{m}>1} s_{\mathrm{m}} \Omega(\mathfrak{I}(1), \mathfrak{I}(\mathrm{m}))}
$$

and $\omega$ is a matrix with $i, j$ th element equal to $\Omega(\mathfrak{I}(\mathrm{i}), \mathfrak{J}(\mathrm{j}))$.

The goal of the margin-based calibration procedure is to minimize the deviation of the implied margins from the actual margins. That is, minimize $D=$ $\left\|\mu^{*}-m^{*}\right\| / \operatorname{sqrt}\left(n_{1}+n_{2}\right)$ by optimizing with respect to $b_{11}$ and the nesting parameters $\left(m^{*}\right.$ has $n_{1}+n_{2}$ elements). The distance criterion $D$ is therefore analogous to a root-mean-squared-error criterion. This procedure gives equal weight to each margin on the assumption that they are equally informative about the structure of the demand model.

${ }^{19}$ Epstein and Rubinfeld (2002) proposed using a coarse grid on the nesting parameters (e.g., $0.75,0.50$, and 0.25 ) to assess the sensitivity of the simulation to different nest structures. The margins provide an alternative approach that calibrates the parameters empirically. 
The analysis of equation (14) depends on the number of nests and margins. We begin with the cases that are likely to be most common and most tractableeither two or three nests. With two nests and two margins there is an exact solution because equation (14) would constitute a system of two equations (for the margins) in two unknowns, the single nesting parameter and the $b_{11}$ coefficient.

With three nests, equation (14) becomes a system of three equations in four unknowns, three nesting parameters, and the $b_{11}$ coefficient. The system is therefore underidentified. The most convenient procedure in this case is to solve (14) by varying one of the nesting parameters exogenously to generate the widest possible range of solutions. Equation (14) places bounds on the solutions because the economically acceptable nesting parameters must lie on the $(0,1]$ interval. Depending on the particular values of the key parameters, these bounds can be fairly tight, resulting in a set of simulations that are still highly informative.

Now consider the possibility of overidentification. The 2-nest system will illustrate. Suppose firm 1 produces brand $A_{11}$ in nest 1 and brand $A_{12}$ in nest 2 . Suppose firm 2 produces a single brand $B_{21}$ that is also located in nest 2 . Equation (14) would constitute a system of three equations for the three known margins, but there are only two unknowns. An exact solution in general does not exist. Instead, we propose using the "minimum distance" estimator $m^{*}$. The solution will not match the observed margins exactly but the deviations should be relatively small if the model is well-specified. If, however, one or more deviations are considered large, we would reevaluate the original nesting assumptions. One possibility in the 2-nest example is that it is more appropriate to place $A_{12}$ in nest 1 . This could be tested by putting $A_{12}$ in a separate nest 3 with the constraints (using the notation in equation (7)) that $\omega_{2}=100 \%$ and $\omega_{3}=$ $\omega_{1}$. A smaller $D$ after again solving equation (14) would provide evidence that the alternative nest structure is preferable. But if the predicted and actual margins still diverged excessively, this could be evidence that $A_{12}$ should be placed in its own nest with independent nesting parameters. ${ }^{20}$ Analogous considerations apply to the 3-nest system.

\section{Measuring Incremental Profit Margins Using ACCOUnTING DATA}

The relevant profit margin to calibrate the model in principle should be based on the profit associated with an incremental change in output, i.e., the difference between the associated incremental revenue and the incremental cost. While

${ }^{20}$ Another possibility, discussed below, is that the nesting assumptions for the brands produced by the non-merging firms are incorrect. 
seemingly straightforward, the empirical determination of economically relevant margins at the brand level raises questions of interpreting the available cost accounting data that are not always resolvable without controversy. Indeed, this has been a relatively neglected area in empirical econometrics. We expect that increasing experience in analyzing margins for use in simulation will lead to further methodological insights and potential consensus on the best way to utilize margin data.

A firm's financial statements for external reporting (e.g., income statements prepared under GAAP) typically are not adequate for deriving margins, implying the need for further accounting and economic analysis. Even internal brand-level data still generally require careful interpretation and possible adjustment. Allocations of common costs of production for multiproduct firms; treatment of depreciation; ${ }^{21}$ classification of costs as fixed or variable (e.g., marketing); and whether to use pre-tax or after-tax profits are some of the major questions likely to arise in practice.

Empirically, two approaches are typically used to determine incremental costs. The first approach relies on a regression analysis in which the dependent variable is total operating costs and the independent variable is either quantity produced or sales revenue. The regression coefficient on the quantity variable provides an estimate of incremental cost. The regression coefficient on the revenue variable is an estimate of 1 minus $\mu$, where $\mu$ is the incremental percentage profit margin. Multiple regression analysis is also used, where the independent variables are various "cost drivers" (e.g., machine hours) that are correlated with output. ${ }^{22}$ Regression is an attractive approach when sufficient data are available, particularly to identify variation in brand-level costs in multiproduct firms. ${ }^{23}$

One potential concern is that the regression may only measure short-run incremental costs, when a longer-run cost measure would be appropriate. For example, constant straight-line depreciation in the dependent variable might not influence the regression coefficient at all. As this example points out, the regression analysis may not provide a complete solution to estimating the economically relevant costs. Additional economic and financial analyses may be necessary to incorporate capacity costs and a return on capital.

The second general approach involves an "account analysis" where each line item in a firm's internal cost reporting system is identified as either "fixed" or

\footnotetext{
${ }^{21}$ Fisher (1987).

${ }^{22}$ Horngren, Dukar, and Foster (2003).

${ }^{23}$ When firms have multiple plants, the regression may require a careful specification with plant-level data. .
} 
"variable." This can be especially useful when the adjustments to cost of goods sold are reasonably straightforward, e.g., subtracting out depreciation or adding in sales commissions. However, account analysis can entail subjective assumptions when the individual line items still combine fixed and variable components. Account analysis can also become arbitrary in the case of multi-product firms, where a variable cost item may have to be allocated across different product lines. This approach also may require separate imputation of an economic return.

The regression and account analysis approaches are likely to be complementary. Some account analysis may be necessary to prepare the input data for the regression. More generally, each method can be used as a check on the other. We note that estimation of incremental costs using both methods is often carried out in the patent damages arena, where the results must survive expert cross-examination. Equally credible analysis should also be feasible in the context of mergers.

We are aware, of course, that econometric estimation of demand elasticities provides an alternative to reliance on accounting data to calibrate the FOCs. While the accounting data present a set of technical problems, one should bear in mind that the margin-based analysis was motivated in large part by the assumption that reliable econometric results were not available for the simulation model. In addition, we think there is separate methodological value in coupling the merger analysis to the type of cost and margin data that is presumably used in actual managerial decision-making.

\section{EXAMPLES}

To illustrate the ideas developed to this point we now discuss several different examples. We begin with model calibration in the 3-firm market example described previously. We then turn to a well-known analysis of the retail beer market. Our last example focuses on identification issues.

\section{A. Calibration in a 3-firm market}

Recall that each of the 3 firms produces a single brand; the shares for the firms are $20 \%, 30 \%$, and $50 \%$, respectively. The industry elasticity is -1 , and the merger partners are firms 1 and 2, with firm 1 having an incremental profit margin of $33.3 \%$.

Suppose first that all brands are assumed to belong in a single nest and that the simulation model is calibrated with the firm 1 margin. PCAIDS generates an elasticity of -2.75 for firm 2 (see Table 1), implying a corresponding margin of $36.4 \%$ (the negative reciprocal of the elasticity). If the actual incremental profit 
margin were close to $36.4 \%$, we would take this as support for the strict proportionality assumption and use of a single nest in the model.

Suppose, however, that firm 2 had a profit margin of $48.1 \%$. This would suggest that firm 2 faces less competition than implied by proportionality. We would infer that the model requires two nests, with firms 1 and 3 in the same nest and firm 2 in a separate nest. The model is exactly identified because the two margins map into the two unknown parameters in equation (14). The solution yields a nesting parameter equal to 0.5 , since (see Table 2) this value for the nesting parameter results in an elasticity for firm 2 of -2.08 , which corresponds to the observed $48.1 \%$ margin.

The decision as to how to group brands into nests is an important one. Thus, firm 2 could also have faced reduced competition if it were in the same nest as firm 3 and firm 1 was in a separate nest. Obviously, the nesting parameter, and the resulting elasticities in the model, can be sensitive to this choice. Beyond the intuition just given, there is one additional helpful guide. A nesting parameter must lie in the interval $(0,1]$ to be economically meaningful. The separate brand 1 nest structure can only satisfy the observed margins if the nesting parameter had the value 2.16, an extraneous solution that in our view rules out this nest from further consideration. We conclude that the most appropriate model should use the nests in Table 2.

We believe it reasonable to utilize a principle of "maximum proportionality" as an additional model selection criterion that is relevant to the grouping problem. When different nest structures are consistent with the margin data and have valid nesting parameters, we advise selecting the structure for which the length of a vector of 1 's minus the vector of feasible nesting parameters is a minimum. This metric yields a solution with a minimum deviation from strict proportionality. ${ }^{24}$ In the case of a single parameter, for example, we would use the nests for which the parameter is closest to 1.0. We view this as an application of Occam's razor, since proportionality has the virtue of simplicity. ${ }^{25}$

${ }^{24}$ A referee has suggested that "maximum proportionality" yields parameters that are most consistent with broad product markets, if these simulations were used for a hypothetical monopolist test per the Horizontal Merger Guidelines. We share this intuition but leave a more detailed investigation for future research.

${ }^{25}$ The assumption of "maximum proportionality" appears consistent with the views of other economists who specialize in merger analysis. See Werden and Froeb (2002), who state that proportionality is "a useful way to define what it means for all goods to be equally close substitutes for each other ... Until reliable contrary evidence is uncovered, we think it sensible to presume that the products of the merging firms are neither especially close nor especially distant substitutes, which means that the IIA property [i.e., proportionality] holds 
Grouping and maximum proportionality in this example are further illustrated in Table 3. For each of the three possible nest configurations, the table shows the ranges of feasible elasticities for firms 1 and 2. More specifically, for each configuration we first hold the elasticity for firm 1 at -3 (the observed $33.3 \%$ margin) and solve for the minimum and maximum possible elasticities (and associated margins) for firm 2 obtained by varying the nesting parameter over $(0,1]$. We then hold the firm 2 elasticity at -2.75 (implied by the firm 1 margin and strict proportionality) and similarly solve for the possible elasticity range for firm 1. That is, each configuration "starts" with a nesting parameter of 1.0 and then maps the feasible deviations from strict proportionality that are consistent with the observed margins. One of the main implications of Table 3 is that the various configurations of nests are able to accommodate an extremely wide range of margins.

When the margins for firms 1 and 2 are $33.3 \%$ and $48.1 \%$, respectively, Table 3 shows that the only feasible nest structure to attain the required elasticities of -3 and -2.08 is Configuration A. The other two nest structures would not be consistent with the margins. Suppose instead that firm 2 had an elasticity of -2.75 (36.4\% margin), but that firm 1 had an elasticity of -1.5 (66.7\% margin). Then $\mathrm{C}$ is the only feasible solution (with a nesting parameter equal to 0.19). Finally, suppose the elasticities for firms 1 and 2 were -3.50 and 2.75, respectively. Configurations $\mathrm{A}$ and $\mathrm{B}$ both provide solutions, with nesting parameters 0.71 and 0.07 , respectively. By maximum proportionality, $\mathrm{A}$ is preferred. A parameter of 0.07 suggests that firm 3 virtually does not compete with firms 1 or 2 . Ideally, other information external to the model would also indicate that firm 3's brand was a reasonably good substitute for firms 1 and 2 to support an inference that 0.71 is reasonable and 0.07 is implausibly small.

approximately." For additional discussion of the IIA assumption in this context see Willig (1991) and the Horizontal Merger Guidelines. 


\section{Table 3}

\section{Nest Configurations and Feasible Elasticities for the Merging Firms}

\begin{tabular}{|c|c|c|c|c|}
\hline \multirow{3}{*}{$\begin{array}{c}\text { Nest } \\
\text { Configuration }\end{array}$} & \multicolumn{2}{|c|}{ Firm 1 Margin $=33.3 \%$} & \multicolumn{2}{|c|}{ Firm 2 Margin $=36.4 \%$} \\
\hline & Implied F & Elasticity: & Implied F & Elasticity: \\
\hline & $\underline{\text { Minimum }}$ & Maximum & Minimum & Maximum \\
\hline A: $(1,3)-(2)$ & -1.02 & -2.75 & -3.00 & -10.00 \\
\hline B: $(1,2)-(3)$ & -2.34 & -2.75 & -2.75 & -3.60 \\
\hline$C:(2,3)-(1)$ & -2.75 & -10.00 & -1.02 & -3.00 \\
\hline
\end{tabular}

Note: Only elasticities in the interval $[-10,-1)$ are included.

\section{B. The Light Beer Market Revisited}

We next analyze a model of retail demand for beer published by Hausman, Leonard, and Zona (1994) ("HLZ"). We focus on the light beer segment that was estimated as part of a multi-stage budgeting model of a broader beer market. HLZ used a panel of weekly store-level data to estimate demand for five different brands (Genesee Light, Coors Light, Old Milwaukee Light, Miller Lite, and Molson Light) using an AIDS model with symmetry and homogeneity restrictions. Although HLZ did not report market shares for the brands, the estimation results contain sufficient information for us to infer reasonable values. For purpose of this example we have assumed the shares shown in Table $4 .{ }^{26}$

Table 4

Estimated Light Beer Market Shares

\begin{tabular}{lc}
\multicolumn{1}{c}{ Brand } & Share $(\%)$ \\
Genesee Lite & 36.6 \\
Coors Light & 25.5 \\
Old Milwaukee Light & 11.5 \\
Miller Lite & 16.0 \\
Molson Lite & 10.4 \\
$\quad$ Total & $100.0 \%$
\end{tabular}

\footnotetext{
${ }^{26}$ It appears that their data were for stores in upstate New York, which (at least several years ago) would account for the brands and relative shares.
} 
We calibrate PCAIDS using HLZ's estimates of an unconditional price elasticity for Genesee of -3.76 and a light beer segment elasticity of -2.424 . These values imply $b_{11}=-0.821$. The resulting PCAIDS coefficients with no nests (using equations (12) and (13) are

$\begin{array}{rrrrr}-0.821 & 0.330 & 0.149 & 0.207 & 0.135 \\ 0.330 & -0.672 & 0.104 & 0.144 & 0.094 \\ 0.149 & 0.104 & -0.360 & 0.065 & 0.042 \\ 0.207 & 0.144 & 0.065 & -0.475 & 0.059 \\ 0.135 & 0.094 & 0.042 & 0.059 & -0.329\end{array}$

with implied elasticities as shown in Table 5.

Table 5

PCAIDS Elasticities (No Nests)

$\begin{array}{crrrrr}\text { Genesee Light } & -3.76 & 0.54 & 0.24 & 0.34 & 0.22 \\ \text { Coors Light } & 0.77 & -4.00 & 0.24 & 0.34 & 0.22 \\ \text { Old Milwaukee Light } & 0.77 & 0.54 & -4.29 & 0.34 & 0.22 \\ \text { Miller Lite } & 0.77 & 0.54 & 0.24 & -4.20 & 0.22 \\ \text { Molson Light } & 0.77 & 0.54 & 0.24 & 0.34 & -4.32\end{array}$

As expected under strict proportionality, the cross-price elasticities in each column are identical. For comparison, Table 6 shows the elasticities estimated by HLZ.

Table 6

HLZ Light Beer Elasticities

$\begin{array}{rrrrrr}\text { Genesee Light } & -3.76 & 0.46 & 0.40 & 0.25 & 0.20 \\ \text { Coors Light } & 0.57 & -4.60 & 0.41 & 0.45 & 0.48 \\ \text { Old Milwaukee Light } & 1.23 & 0.96 & -6.10 & 0.84 & 0.57 \\ \text { Miller Lite } & 0.51 & 0.74 & 0.59 & -5.04 & 0.58 \\ \text { Molson Light } & 0.68 & 1.21 & 0.61 & 0.89 & -5.84 \\ & & & & & \\ \begin{array}{c}\text { Ave. HLZ cross price } \\ \text { elasticity }\end{array} & 0.75 & 0.84 & 0.50 & 0.61 & 0.46\end{array}$

The variation in the HLZ cross-price elasticities suggests that strict proportionality is not satisfied within a segment defined as these five brands. (In 
contrast, HLZ's results support proportionality much more strongly in their premium beer segment.)

Deviating from proportionality requires assumptions about nesting. While beer lovers can debate what brands are the closest substitutes, HLZ suggest that Coors Light and Miller Lite are particularly close. We put these brands in nest 1, along with Molson Light as another high quality, heavily advertised national brand. We find it plausible that Old Milwaukee Light occupies a middle ground between those three brands and Genesee Light, a regional label that is not without its charms. Accordingly, we put them in separate nests 2 and 3, respectively. Finally, we hypothesize the following nesting parameters:

$$
\Omega=\left(\begin{array}{lll}
100 \% & 75 \% & 50 \% \\
75 \% & 100 \% & 75 \% \\
50 \% & 75 \% & 100 \%
\end{array}\right) .
$$

The off-diagonal elements reflect moderate departures from proportionality. Nest 2 is "equally far" from nests 1 and 3 and nest 3 is relatively farther from nest 1 .

Table 7 shows the result of recalibrating PCAIDS with these additional parameters. This structure brings the PCAIDS elasticities quite close to the unrestricted AIDS structure. That is, it appears that the four PCAIDS parameters ( $b_{11}$ and three nesting parameters) contain essentially the same information as the 25 coefficients estimated by HLZ.

Table 7

PCAIDS Elasticities (3 Nests)

$\begin{array}{crrrrr}\text { Genesee Light } & -3.76 & 0.46 & 0.40 & 0.29 & 0.19 \\ \text { Coors Light } & 0.67 & -4.82 & 0.40 & 0.81 & 0.53 \\ \text { Old Milwaukee Light } & 1.26 & 0.88 & -5.47 & 0.55 & 0.36 \\ \text { Miller Lite } & 0.67 & 1.29 & 0.40 & -5.30 & 0.53 \\ \text { Molson Light } & 0.67 & 1.29 & 0.40 & 0.81 & -5.58 \\ & & & & & \\ \text { Average PCAIDS cross- } & 0.81 & 0.98 & 0.40 & 0.62 & 0.40\end{array}$




\section{Calibration in a 3-Nest System}

Suppose there are five firms in the market, the merger partners (A, B) plus three competitors. Firm A sells two brands. The other firms sell one brand. There are three nests (Popular, Budget, and Prestige). Collectively, firms A and B sell into all three nests. In the absence of nests, we would calibrate the PCAIDS model if we knew one brand elasticity. Now, however, we have the case of 3 FOC equations (for the 3 brands produced by the merger partners) and 4 unknowns ( $b_{11}$ and the three nesting parameters). The shares and nests in the market are indicated in Table 8.

Table 8

Shares in a 3 Nest Market

\begin{tabular}{|c|c|c|}
\hline Firm-Brand & Share $(\%)$ & Nest \\
\hline A-1 & 10.0 & Popular \\
\hline A-2 & 7.5 & Prestige \\
\hline B & 12.5 & Budget \\
\hline $\mathrm{C}$ & 15.0 & Popular \\
\hline D & 25.0 & Budget \\
\hline $\mathrm{E}$ & 30.0 & Popular \\
\hline Total & 100.0 & \\
\hline
\end{tabular}

We consider three different margin scenarios to create three different examples of nest calibration.

\section{Scenario 1}

Suppose the margins for brands A-1, A-2, and B are $40.0 \%, 55.0 \%$, and $45.0 \%$, respectively, and the overall market demand elasticity is -1.0 . First, we test whether the 3 margins can be explained with only 2 nests. This is done by solving equation (14) three times, each time fixing a different $\omega_{1}$ at $100 \%$. We found that in no case was there a feasible solution. That is, each solution to equation (14) with $\omega_{1}=100 \%$ required at least one nesting parameter outside $(0,1]$ to satisfy the FOCs. This is an important piece of information that supports the necessity of using a model specification with at least three nests.

We next find the range of feasible solutions for equation (14) by using a coarse grid to set $\omega_{1}$ exogenously. We found that a value of approximately 0.75 was the highest value for $\omega_{1}$ that permitted a feasible solution. We then solved equation (14) three more times, setting $\omega_{1}$ alternately to $0.50,0.25$, and 0.01 . 
Each exogenous value for $\omega_{1}$ implies corresponding unique values for $\omega_{2}$ and $\omega_{3}$. Table 9 reports the range of solutions.

\section{Table 9}

\section{Nesting Parameters Consistent with Margins of 40\%, 55\%, and 45\%}

\begin{tabular}{ccccc} 
& \multicolumn{3}{c}{ Nesting Parameter Vector } \\
Case & Budget_Prestige & Budget_Popular & Popular-Prestige \\
a) & $\omega_{1}$ & $\omega_{2}$ & $\omega_{3}$ \\
b) & 0.75 & 0.24 & 0.03 \\
c) & 0.50 & 0.30 & 0.24 \\
d) & 0.25 & 0.36 & 0.46 \\
d & 0.01 & 0.41 & 0.66
\end{tabular}

The results show a fairly tight range for $\omega_{2}$, the Budget-Popular nesting parameter, centered at approximately 0.32 . While each of the four combinations is consistent with the observed margins, other considerations may point to one of them as the most appropriate. For example, evidence that Popular is substitutable for Prestige may rule out case a) because $\omega_{3}=0.03$ would be too small and therefore extraneous. It may also be reasonable to restrict attention to parameterizations in which neighboring quality levels were relatively more substitutable for each other than quality levels that are separated by a third level. ${ }^{27}$ For example, BMW (Prestige) should be more substitutable for Camry (Popular) than Ford Escort (Budget). The implication in this instance would be to require $\omega_{1}<\omega_{3}$, which would further rule out case b). Of the two remaining candidates, case c) would be preferred by maximum proportionality but evidence of lack of Prestige-Budget competition could justify case d). The key point is that the plausible nesting parameters have fairly tight bounds, in the neighborhood of $(0.13,0.37,0.56)$, using the mid-points between the corresponding values from c) and $\mathrm{d}$ ). This type of procedure should often determine the nesting parameters to an acceptable level of precision. Of course, in practice, one can perform simulations using the alternative calibrations to test the sensitivity of the results to a particular choice of parameters.

\section{Scenario 2}

Suppose the margins now are $40.0 \%, 35.0 \%$, and $35.0 \%$. In this case, there is no feasible solution for any set of nesting parameters in the $(0,1]$ range. There are several possible explanations. First, the margins or the market price elasticity

${ }^{27}$ We thank one of the referees for this suggestion. 
may not have been measured accurately. Second, if firms are not Bertrand pricers or markets are not in equilibrium then the assumptions of the model would not apply. A third possibility - the one that we find the most intriguing - is that the assignment of the brands to the nests was not reasonable. For example, there is a feasible solution if $\mathrm{C}$ is moved from Popular to Prestige. This suggests that using margin data with PCAIDS can offer a useful methodology for model specification purposes.

\section{Scenario 3}

Finally, suppose the margins are $40.0 \%$ for all three brands. There is a feasible solution in this case. Moreover, the range of feasible solutions is tight, as Table 10 illustrates.

Table 10

\section{Nesting Parameters Consistent with $40 \%$ Margins for All Brands}

$\begin{array}{ccc}\text { Budget_Prestige } & \text { Budget_Popular } & \text { Popular_Prestige } \\ \omega_{1} & \omega_{2} & \omega_{3} \\ 1.00 & 0.71 & 0.76 \\ 0.80 & 0.80 & 1.00 \\ 0.99 & 0.72 & 0.78\end{array}$

The nesting parameters are not identified, but are nevertheless estimated with a high level of precision.

\section{CONCLUDING REMARKS}

We believe that the direct use of brand-margin data to estimate the parameters of demand systems offers a fruitful empirical basis for merger simulation analysis. We have shown, within the PCAIDS framework, that the margins serve to calibrate empirically the nest structure (groups of products that are particularly close substitutes) and generalize the analysis of own- and cross-price elasticities. PCAIDS in its basic formulation relies on an assumption of "proportionality" which greatly reduces the number of free parameters but constrains all cross-price elasticities corresponding to a given price change to be equal. By incorporating nests, however, PCAIDS can relax the proportionality constraint and more closely approximate an unconstrained AIDS model.

This modeling strategy exploits information that is likely to be available in the typical merger investigation (at least for the merging parties), but that has not 
been integrated into existing econometric models used for merger simulation. In some cases, the nesting parameters will be exactly identified. In other cases, they will be underidentified, but simulated price effects can be still bounded. In still other cases, the nesting parameters will be overidentified, which permits an analysis of the robustness of the underlying demand specification.

PCAIDS with nests may be especially appealing for retail mergers where scanner data do not exist and for wholesale mergers. Available brand margin data, market shares, and an estimate of the market demand elasticity should be sufficient to make PCAIDS feasible in such cases. In contrast, it may be difficult or even impossible to implement more data-intensive methodologies that rely on the econometric estimation of demand systems. Some of the areas for continued work include: how does PCAIDS compare in practice and in theory to a logit model with nests? to a multi-stage AIDS model? What are the most appropriate procedures for testing and evaluating the robustness of the PCAIDS results to the choice of nesting parameters? These and other questions are ripe for future research. 


\section{BIBLIOGRAPHY}

Alston, Julian M., Kenneth A. Foster, and Richard D. Green (1994), "Estimating Elasticities with the Linear Approximate Almost Ideal Demand System: Some Monte Carlo Results," 76 Rev. Econ. Stat. 351-356.

Baker, Jonathan and Daniel L. Rubinfeld (1999), "Empirical Methods in Antitrust: Review and Evidence," 1 Am. Law and Econ. Rev. 386-435.

Berry, Steven., James Levinsohn, and Ariel Pakes (1995), "Automobile Price in Market Equilibrium," 63 Econometrica 841-90.

Blattberg, Robert and Kenneth Wisniewski (1989), "Price-Induced Patterns of Competition,” 8 Marketing Science 291-309.

Deaton, Angus and John Muellbauer (1980), "An Almost Ideal Demand System," 70 Am. Econ. Rev. 312-326.

Epstein, Roy J. and Daniel L. Rubinfeld (2002), "Merger Simulation: A Simplified Approach with New Applications," 69 Antitrust L. J. 883-919.

Fisher, Franklin M. (1987) "On the Misuse of the Profits-Sales Ratio to Infer Monopoly Power” 18 RAND J. of Econ. 384-396.

Horngren, Charles T., Srikant M. Dukar, and George Foster (2003), Cost Accounting: A Managerial Emphasis, 11th edition, Upper Saddle River, NJ: Prentice-Hall, Chapter 10.

Hausman, Jerry, Gregory Leonard, and J. Douglas Zona, (1994), "Competitive Analysis with Differenciated Products," 34 Annales D'Économie et de Statistique 159-180.

Pinske, Joris and Margaret E. Slade, "Mergers, Brand Competition, and the Price of a Pint," European Econ. Rev. forthcoming (as of Jan. 2004).

Werden, Gregory J. and Luke M. Froeb (1994), "The Effects of Mergers in Differentiated Products Industries: Logit Demand and Merger Policy," $10 \mathrm{~J}$. of Law, Econ., and Org. 407-426.

Werden, Gregory J. and Luke M. Froeb (2002), "The Antitrust Logit Model for Predicting Unilateral Competitive Effects," 70 Antitrust L. J. 257-260.

Willig, Robert D. (1991), "Merger Analysis, Industrial Organization Theory, and Merger Guidelines," Brookings Papers On Economic Activity, Microeconomics. 281-312. 
Wu, Lawrence (2003), "Two Methods of Determining Elasticities of Demand and Their Use in Merger Simulation," NERA Antitrust Insights, available from www.nera.com. 